\title{
An analysis on overall crack-number-density of short-fatigue-cracks
}

\author{
Youshi Hong *, Yu Qiao \\ Lab for Non-linear Mechanics of Continuous Media, Institute of Mechanics, Chinese Academy of Sciences, 15 Zhongguancun Road, \\ Beijing 100080, People's Republic of China
}

Received 18 August 1997; received in revised form 12 March 1999

\begin{abstract}
The evolution of dispersed short-fatigue-cracks is analysed based on the equilibrium of crack-number-density (CND). By separating the mean value and the stochastic fluctuation of local CND, the equilibrium equation of overall CND is derived. Comparing with the mean-field equilibrium equation, the equilibrium equation of overall CND has different forms in the expression of crack-nucleation-rate or crack-growth-rate. The simulation results are compared with experimental measurements showing the stochastic analyses provide consistent tendency with experiments. The discrepancy in simulation results between overall CND and mean-field CND is discussed. (c) 1999 Elsevier Science Ltd. All rights reserved.
\end{abstract}

Keywords: Short fatigue cracks; Crack number density; Collective damage; Stochastic analyses

\section{Introduction}

Generally, the fatigue process of metallic materials can be identified as two stages: short-crack regime and long-crack regime (Hussain et al., 1993; Fang et al., 1995). In the short-crack regime, cracks keep nucleating and growing within grain domains, which seldom may overcome grainboundary obstacles and develop to be crossboundary cracks. With the progress of the fatigue process, the crack-nucleation-rate keeps decreasing for cracks approaching grain boundaries and a few short-fatigue-cracks may grow into neighbour grains. The appearance of a crack with a size large enough to overcome grain-boundary obstacle ef-

\footnotetext{
${ }^{*}$ Corresponding author. Tel.: +86 106261 3730; fax: +86 10 6256 1284; e-mail: hongys@lnm.imech.ac.cn
}

fect characterises the end of the short-crack regime and the beginning of the long-crack regime. Since the short-crack regime usually takes up a large portion of fatigue life, therefore it is in engineering important to investigate the fatigue damage due to short crack evolution, so as to assess the reliability and fatigue life of material under cyclic loading.

During the fatigue damage process, the initiation and the growth of short cracks are always randomly distributed. In some local areas, short cracks may densely appear; simultaneously, there may exist some other areas even without any short crack damage. This suggests that the collective damage of short fatigue cracks is a stochastic feature, which calls for analyses to get a better understanding for such a process.

In the present paper, we describe the method of crack-number-density (CND) analysis which is proposed to deal with the collective crack 
evolution problem. A stochastic consideration is introduced for both local field and overall field derivations. The simulation results of stochastic analyses and mean-field theory are obtained, and compared with experimental measurements. The discrepancy between stochastic analyses and mean-field theory is discussed.

\section{Model of crack-number-density analysis}

Considering the collective characteristics existing in short-crack regime, one may introduce crack-number-density (CND) to analyse fatigue damage (Fang et al., 1995; Qiao and Hong, 1998a). Omitting the difference in the propensity of short cracks at separate local areas of material, we write the non-dimensional mean-field equilibrium equation of CND as

$\frac{\partial}{\partial t} \bar{n}^{\prime}(c, t)+\frac{\partial}{\partial c}\left[\bar{A}^{\prime}(c) \bar{n}^{\prime}(c, t)\right]=N_{\mathrm{g}} \bar{n}_{\mathrm{N}}^{\prime}(c)$,

where $\bar{n}^{\prime}(c, t)$ is mean-field CND, with $\bar{n}^{\prime}(c, t) \mathrm{d} c$ being the number of cracks with length between $c$ and $c+\mathrm{d} c$ at time $t ; \bar{A}^{\prime}(c)$ is average crack-growthrate; and $\bar{n}_{\mathrm{N}}^{\prime}(c)$ is average crack-nucleation-rate. All of these physical quantities are normalized and the non-dimensional coefficient $N_{\mathrm{g}}=$ $\left(n_{\mathrm{N}}^{*} d\right) /\left(n^{*} A^{*}\right)$, with $n_{\mathrm{N}}^{*}$ being the characteristic crack-nucleation-rate, $A^{*}$ the characteristic crackgrowth-rate, $n^{*}$ the characteristic CND and $d$ the characteristic dimension of the material concerned (e.g. the grain diameter). Eq. (1) describes the equilibrium of CND in phase space. The second term on the left side describes the flow of CND, which is attributed to crack growth; and the term on the right side describes the contribution to CND made by crack nucleation.

The evolution process of mean-field CND was derived by solving Eq. (1) and thus the relevant damage parameters, such as the maximum crack length and the total number of short-fatiguecracks, were discussed (Fang et al., 1995; Qiao and Hong, 1998a; Hong and Qiao, 1998). These damage parameters were introduced to illustrate the extent of material damage. Fig. 1 shows a numerical result of the mean-field equilibrium equation. Through the figure we see the dual-peak

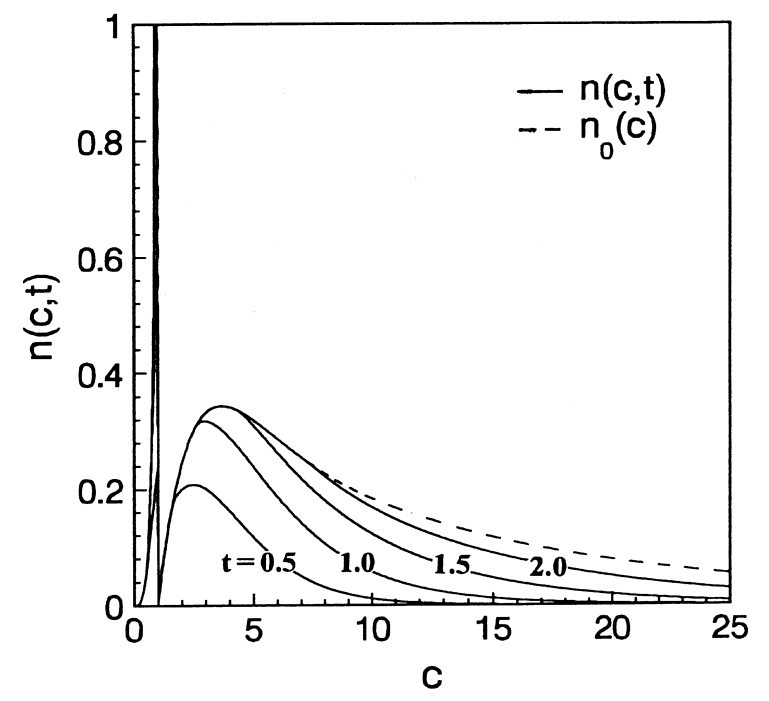

Fig. 1. Numerical result for CND evolution of mean-field theory, dashed curve representing the saturation distribution. $A_{\mathrm{d}}=0$ and $\bar{d}=1$ were used in the calculation.

distribution of CND, which is in agreement with the dual-peak feature observed in experiments (Fang et al., 1995). Note that the above analyses are focused on mean-field case, i.e. different parts of material are assumed to have the same local mechanical characteristics. However, in the real fatigue damage process, there always exists stochastic fluctuation of local area behaviour in material (Price, 1988; Hong et al., 1989, 1991).

Considering the difference in local areas of material, we introduced the concept of local CND and obtained the equilibrium equation of local CND (Qiao and Hong, 1998b):

$$
\begin{aligned}
& \frac{\partial}{\partial t} n(c, t, \vec{x})+\frac{\partial}{\partial c}[A(c, t, \vec{x}) n(c, t, \vec{x})] \\
& \quad=N_{\mathrm{g}} n_{\mathrm{N}}(c, t, \vec{x}),
\end{aligned}
$$

where $\vec{x}$ is the position of a small area of material, $n(c, t, \vec{x}), A(c, t, \vec{x})$ and $n_{\mathrm{N}}(c, t, \vec{x})$ are local CND, local crack-growth-rate and local crack-nucleation-rate, respectively. The local area is treated as small enough to be considered as a point at macroscale. On the other hand, the local area also contains enough short-fatigue-cracks so that the concept of CND can be applied. It is observed in 
experiments that a viewed area on specimen surface may comprise a continuous low-damage-extent area and dispersed high-damage-extent areas (Price, 1988; Hong et al., 1997). Fig. 2 is a photograph showing the uneven distribution of short fatigue cracks. This was taken from the iso-stress specimen of a low carbon steel subjected to the fatigue loading of stress ratio $R=-1$, frequency $f=11 \mathrm{~Hz}$ and $\sigma_{\max }=1.17 \sigma_{\mathrm{y}}$, where $\sigma_{\max }$ is the specimen surface maximum stress and $\sigma_{\mathrm{y}}$ is the yield stress of the material. Fig. 2 is a typical example that during the progression of fatigue damage, within some grain domains, short cracks have become densely distributed, whereas some other ferrite domains remain undamaged without a single short crack appearing. The experimental process and the results have been described elsewhere (Hong et al., 1989, 1991, 1997). The stochastic appearance of short crack distribution is schematically illustrated in Fig. 3. Considering that the interaction of short-fatigue-cracks is weak in low-damage-extent area, we assume that local areas are independent of each other. By solving the equilibrium equation of local CND, and taking into account the stochastic fluctuation of local crack-growth-rate and local crack-nucleation-rate, the damage evolution in different small areas of material was studied. According to experimental

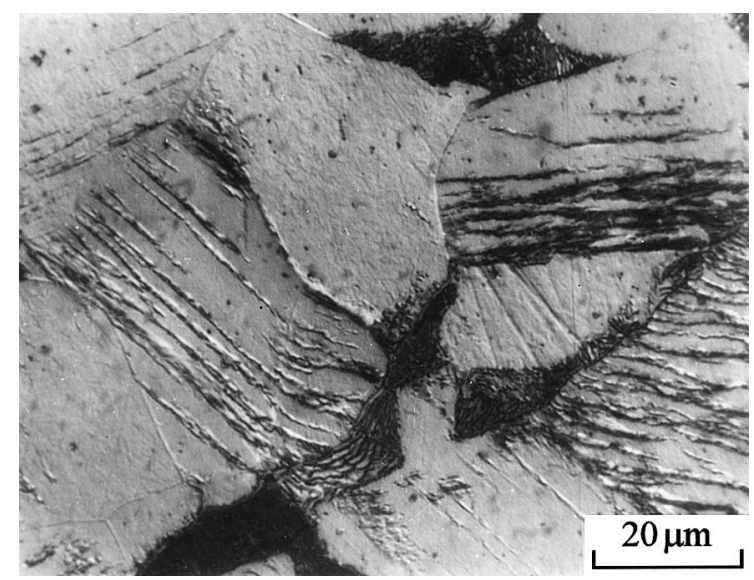

Fig. 2. Photograph showing short-fatigue-cracks developed in some ferrite grains and without crack damage in other ferrite domains, vertical direction parallel to tensile stress.

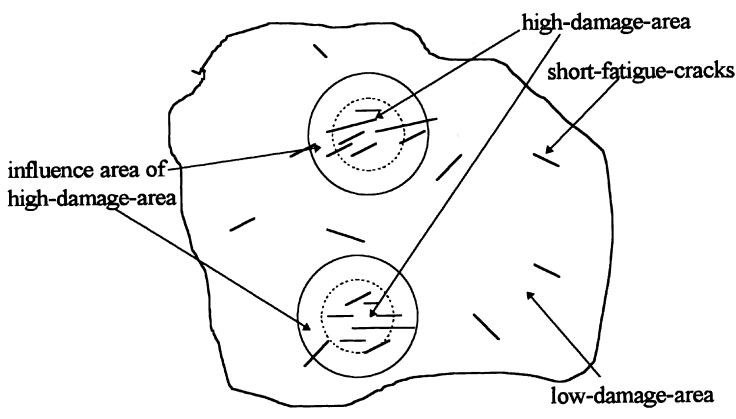

Fig. 3. Schematic illustration of dispersed short-fatigue-cracks.

observations, we assumed that local crack-growthrate and local crack-nucleation-rate have the following forms, respectively (Qiao and Hong, 1998b):

$A(c, t, \vec{x})=\left[A_{0}(c)+L(c) W_{1}(\vec{x})\right]\left[\alpha D_{0 l}^{\beta}(t, \vec{x})\right]$,
$n_{\mathrm{N}}(c, t, \vec{x})=\left[n_{\mathrm{N} 0}(c)+L^{*}(c) W_{2}(\vec{x})\right]\left[p D_{0 l}^{q}(t, \vec{x})\right]$,

where $A_{0}(c)$ and $n_{\mathrm{N} 0}(c)$ are respectively the mean values of local crack-growth-rate and local cracknucleation-rate; $D_{0 l}$ is number of cracks in local area; $\alpha, \beta, p$ and $q$ are material parameters; $L$ and $L^{*}$ are functions respectively illustrating stochastic fluctuation of $A$ and $n_{\mathrm{N}}$; and $W_{1}$ and $W_{2}$ are two white-noise process. Local CND was derived from Eq. (2), and thus local maximum crack length and local number of cracks were obtained. Overall maximum crack length and overall number of cracks were calculated by analysing the local damage evolution in each local area. Fig. 4 shows the results of overall maximum crack length $c_{\max }$ against normalized time $t$, where the datum points are the results of 10 stochastic numerical simulations with the dashed line being the regressed curve of the stochastic results and the solid line is the numerical result of mean-field theory (Eq. (1)). The difference in the results between mean-field equilibrium equation and the stochastic analyses becomes evident when $t$ is beyond 1.6. In other words, the result of stochastic analyses is almost consistent with that of mean-field equation at the initial stage of damage evolution process and it becomes smaller than the mean-field 


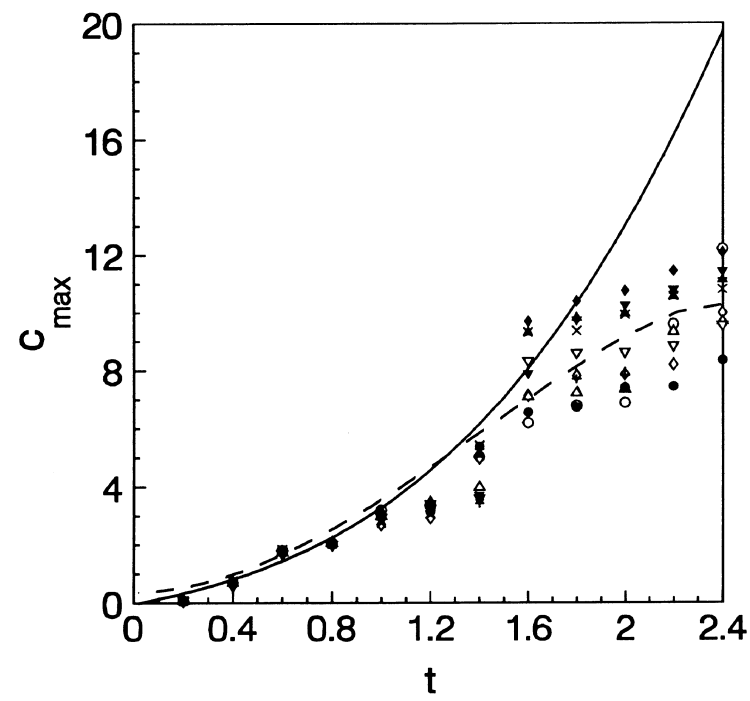

Fig. 4. Results of overall maximum crack length against time derived by mean-field theory and stochastic analyses. Data points showing results of stochastic simulations with dashed line as regressed curve and solid line being the result of meanfield theory.

value at a certain stage of the fatigue evolution process.

Fig. 5 shows the result of overall maximum crack length $c_{\max }$ versus overall number of cracks $D_{0}$, where the solid curve representing the result of mean field theory and data points representing the result of stochastic simulations. It is seen that the relationship between $c_{\max }$ and $D_{0}$ has the same trend for mean field theory and stochastic analysis. Fig. 5 also indicates that $c_{\max }$ and $D_{0}$ are approximately linearly correlated.

\section{Analyses of overall CND}

\subsection{Evolution equation of overall CND}

The short-crack damage evolution is considered an ergodic system. Therefore, the equilibrium equation of overall CND can be derived by integrating the equilibrium equation of local CND. Let $\bar{n}(c, t)$ be CND in a unit area of material. According to the definition of local CND $n(c, t, \vec{x})$, we have:

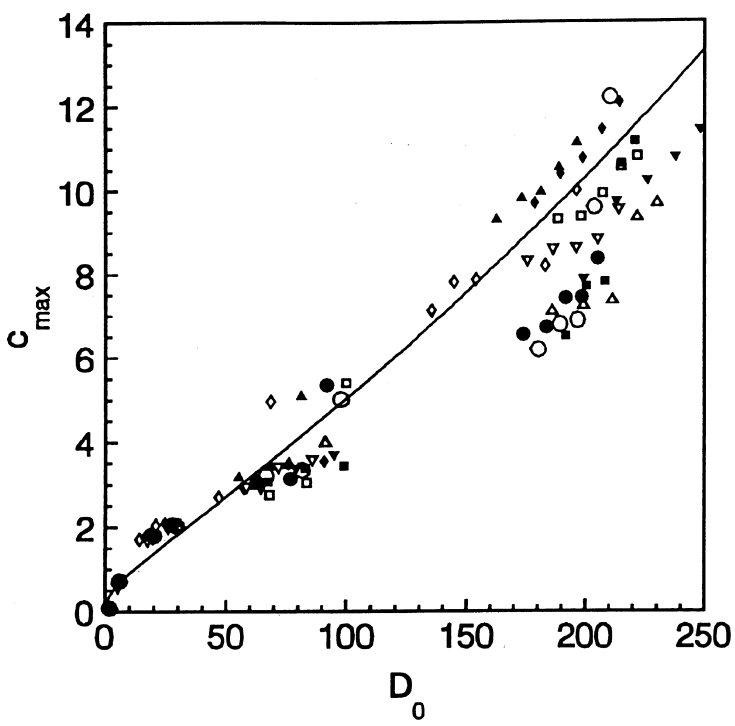

Fig. 5. Relationship between whole field maximum crack size and total number of short cracks, solid curve representing result of mean-field theory, data points representing result of stochastic simulations.

$\bar{n}(c, t)=\frac{1}{S} \int_{\Omega} n(c, t, \vec{x}) \mathrm{d} \vec{x}$,

where $S$ is the area of integration part $\Omega$ on specimen surface. If a unit area is regarded as a local area, then $\bar{n}(c, t)$ is just the average CND. It follows that

$n(c, t, \vec{x})=\bar{n}(c, t)+\tilde{n}(c, t, \vec{x})$,

where $\tilde{n}(c, t, \vec{x})$ is the stochastic fluctuation of local CND $n(c, t, \vec{x})$. Local crack-growth-rate $A(c, t, \vec{x})$ and local crack-nucleation-rate $n_{\mathrm{N}}(c, t, \vec{x})$ can also be written as:

$A(c, t, \vec{x})=\bar{A}(c, t)+\tilde{A}(c, t, \vec{x})$,

$n_{\mathrm{N}}(c, t, \vec{x})=\bar{n}_{\mathrm{N}}(c, t)+\tilde{n}_{\mathrm{N}}(c, t, \vec{x})$,

where $\bar{A}(c, t)$ and $\bar{n}_{\mathrm{N}}(c, t)$ are respectively the mean values of $A(c, t, \vec{x})$ and $n_{\mathrm{N}}(c, t, \vec{x})$, and $\tilde{A}(c, t, \vec{x})$ and $\tilde{n}_{\mathrm{N}}(c, t, \vec{x})$ are respectively the stochastic fluctuations of $A(c, t, \vec{x})$ and $n_{\mathrm{N}}(c, t, \vec{x})$. Substituting Eqs. (6)-(8) into Eq. (2) and integrating in $\Omega$, we have 


$$
\begin{aligned}
& \frac{\partial \bar{n}(c, t)}{\partial t}+\frac{1}{S} \int_{\Omega} \frac{\partial \tilde{n}(c, t, \vec{x})}{\partial t} \mathrm{~d} \vec{x} \\
& \quad+\frac{\partial[\bar{A}(c, t) \bar{n}(c, t)]}{\partial c}+\frac{1}{S} \int_{\Omega} \frac{\partial[\bar{A}(c, t) \tilde{n}(c, t, \vec{x})]}{\partial c} \mathrm{~d} \vec{x} \\
& \quad+\frac{1}{S} \int_{\Omega} \frac{\partial[\tilde{A}(c, t, \vec{x}) \bar{n}(c, t)]}{\partial c} \mathrm{~d} \vec{x} \\
& \quad+\frac{1}{S} \int_{\Omega} \frac{\partial[\tilde{A}(c, t, \vec{x}) \tilde{n}(c, t, \vec{x})]}{\partial c} \mathrm{~d} \vec{x} \\
& =N_{\mathrm{g}} \bar{n}_{\mathrm{N}}(c, t)+\frac{1}{S} \int_{\Omega} N_{\mathrm{g}} \tilde{n}_{\mathrm{N}}(c, t, \vec{x}) \mathrm{d} \vec{x} .
\end{aligned}
$$

Note that for an ergodic system

$$
\begin{aligned}
& \int_{\Omega} \tilde{n}(c, t, \vec{x}) \mathrm{d} \vec{x}=0, \\
& \int_{\Omega} \tilde{A}(c, t, \vec{x}) \mathrm{d} \vec{x}=0, \\
& \int_{\Omega} \tilde{n}_{\mathrm{N}}(c, t, \vec{x}) \mathrm{d} \vec{x}=0 .
\end{aligned}
$$

Substitution of Eqs. (10)-(12) into Eq. (9) gives

$$
\begin{aligned}
& \frac{\partial \bar{n}(c, t)}{\partial t}+\frac{\partial[\bar{A}(c, t) \bar{n}(c, t)]}{\partial c} \\
& \quad=N_{\mathrm{g}} \bar{n}_{\mathrm{N}}(c, t)-\left(\frac{\partial[\tilde{A}(c, t, \vec{x}) \tilde{n}(c, t, \vec{x})]}{\partial c}\right),
\end{aligned}
$$

where

$$
\begin{aligned}
& \overline{\left(\frac{\partial[\tilde{A}(c, t, \vec{x}) \tilde{n}(c, t, \vec{x})]}{\partial c}\right)} \\
& =\frac{1}{S} \int_{\Omega}\left\{\frac{\partial[\tilde{A}(c, t, \vec{x}) \tilde{n}(c, t, \vec{x})]}{\partial c}\right\} \mathrm{d} \vec{x} .
\end{aligned}
$$

Eq. (13) is the equilibrium equation of overall CND, which shows the equilibrium of overall
CND in phase space with local CND fluctuating in different local areas.

\subsection{Fluctuation influence term}

Comparing Eq. (13) with Eq. (1), we see that there appears, in the equilibrium equation of overall CND, a new term $-\overline{(\partial[\tilde{A}(c, t, \vec{x}) \tilde{n}(c, t, \vec{x})] /(\partial c))}$, which we call the fluctuation influence term (FIT) in the following. FIT represents the effects of the stochastic fluctuation of local damage on overall damage and it is caused by the non-linear responses in the damage evolution process. The comparison of Eq. (13) with Eq. (1) suggests that FIT is the cause of the deviation of stochastic analyses from mean-field theory shown in Fig. 4.

Referring to the definition of Eq. (14), we may write FIT as

$$
\begin{aligned}
& -\overline{\left(\frac{\partial[\tilde{A}(c, t, \vec{x}) \tilde{n}(c, t, \vec{x})]}{\partial c}\right)} \\
& =-\frac{\partial}{\partial c}\left[\frac{1}{S} \int_{\Omega} \tilde{A}(c, t, \vec{x}) \tilde{n}(c, t, \vec{x}) \mathrm{d} \vec{x}\right] .
\end{aligned}
$$

According to Eqs. (10) and (11), the mean values of $\tilde{A}(c, t, \vec{x})$ and $\tilde{n}(c, t, \vec{x})$ are zero. Thus the function in the brackets on the right side of above equation is the covariance of $\tilde{A}(c, t, \vec{x})$ and $\tilde{n}(c, t, \vec{x})$. Here we write it as $\mu(c, t)$. In general,

$\mu(c, t)=\rho(c, t) \sigma_{A}(c, t) \sigma_{n}(c, t)$,

where $\sigma_{A}(c, t)$ is the standard deviation of local crack-growth-rate $A(c, t, \vec{x}) ; \sigma_{n}(c, t)$ is the standard deviation of local CND $n(c, t, \vec{x})$ and $\rho(c, t)$ is the correlation coefficient between $A(c, t, \vec{x})$ and $n(c, t, \vec{x})$. Substituting Eqs. (15) and (16) into (13), the equilibrium equation of overall CND becomes:

$$
\begin{aligned}
& \frac{\partial \bar{n}(c, t)}{\partial t}+\frac{\partial[\bar{A}(c, t) \bar{n}(c, t)]}{\partial c} \\
& \quad=N_{\mathrm{g}} \bar{n}_{\mathrm{N}}(c, t)-\frac{\partial \mu(c, t)}{\partial c} \\
& \quad=N_{\mathrm{g}} \bar{n}_{\mathrm{N}}(c, t)-\frac{\partial}{\partial c}\left[\rho(c, t) \sigma_{A}(c, t) \sigma_{n}(c, t)\right] .
\end{aligned}
$$


One may notice that if $|\rho(c, t)|=0$, Eq. (17) reduces to the form of Eq. (1) and the result of stochastic analyses is the same with that of meanfield theory.

In order to discuss the response of FIT with the evolution equation of overall CND, we define the effective crack-growth-rate as

$$
\begin{aligned}
\hat{A}(c, t) & =\frac{\overline{A(c, t, \vec{x}) n(c, t, \vec{x})}}{\bar{n}(c, t)} \\
& =\frac{\bar{A}(c, t) \bar{n}(c, t)+\overline{\tilde{A}(c, t, \vec{x}) \tilde{n}(c, t, \vec{x})}}{\bar{n}(c, t)},
\end{aligned}
$$

where

$\overline{A(c, t, \vec{x}) n(c, t, \vec{x})}=\frac{1}{S} \int_{\Omega} A(c, t, \vec{x}) n(c, t, \vec{x}) \mathrm{d} \vec{x}$.

Substitution of Eq. (18) into (13) gives another form of the equilibrium equation of overall CND:

$\frac{\partial \bar{n}(c, t)}{\partial t}+\frac{\partial[\hat{A}(c, t) \bar{n}(c, t)]}{\partial c}=N_{\mathrm{g}} \bar{n}_{\mathrm{N}}(c, t)$.

Eq. (19) implies that the deviation of stochastic analyses from mean-field theory may also be attributed to the difference between effective crackgrowth-rate $\hat{A}(c, t)$ and average crack-growth-rate $\bar{A}(c, t)$.

\subsection{Comparison with experimental results}

Our previous experimental investigation of a low carbon steel indicated that the progression of short-fatigue-cracks is a collective evolution process with the gradual increase in crack number per unit area with increasing number of fatigue cycles (Hong et al., 1997). Our recent experimental work again revealed the collective damage evolution of short fatigue cracks for a stainless steel. Fig. 6 shows the variation of the total number of short cracks $D_{0}$ with the normalized number of fatigue cycles $N / N_{\mathrm{f}}$ for two kinds of specimen conditions with average grain sizes of 72 and $207 \mu \mathrm{m}$. The two data sets give a similar trend between $D_{0}$ and $N / N_{\mathrm{f}}$ in spite of their large difference in grain size. The determinations also indicated that the short crack regime takes up as much as $87 \%$ of total fatigue life. The detailed

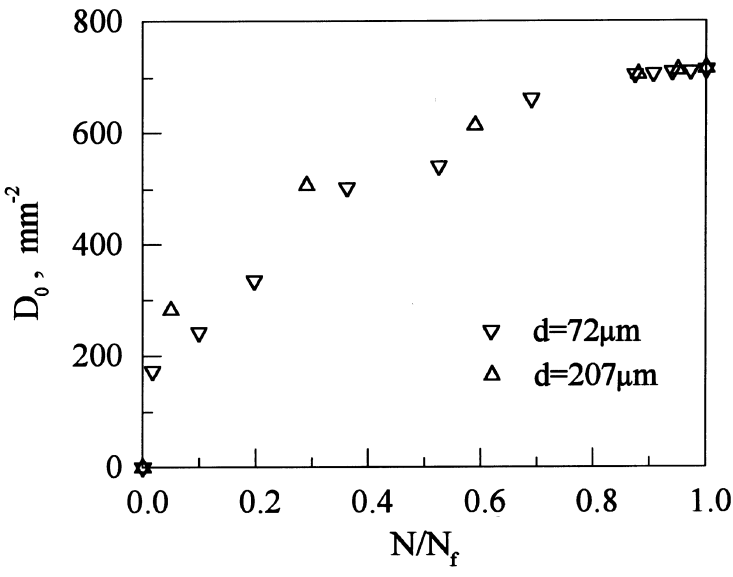

Fig. 6. Total number of short cracks $D_{0}$ versus normalized number of fatigue cycles $N / N_{\mathrm{f}}$ for a stainless steel with two grades of grain size.

procedure and results were reported recently (Hong et al., 1998, 1999).

Referring to the linear correlation between $c_{\max }$ and $D_{0}$ indicated by Fig. 5 and noting that the normalized time $t$ is associated with normalized fatigue cycles $N / N_{\mathrm{f}}$, one may evaluate that the variation trend of $c_{\max }$ with $t$ is equivalent to that of $D_{0}$ with $N / N_{\mathrm{f}}$. Thus, we see the evidence that the variation tendency between $c_{\max }$ and $t$ given by stochastic simulations shown in Fig. 4 is consistent with that of the experimental results shown in Fig. 6. The solid curve, plotted in Fig. 4 obtained by mean-field theory, overestimates the collective short crack evolution when $t$ tends to a large value.

\section{Effect of fluctuation influence term}

Subtracting Eq. (13) from Eq. (2) gives the evolution equation for stochastic fluctuation of local CND:

$$
\begin{aligned}
& \frac{\partial \tilde{n}(c, t, \vec{x})}{\partial t}+\frac{\partial}{\partial c}[\bar{A}(c, t) \tilde{n}(c, t, \vec{x})+\tilde{A}(c, t, \vec{x}) \bar{n}(c, t)] \\
& \quad=N_{\mathrm{g}} \tilde{n}_{\mathrm{N}}(c, t, \vec{x}) .
\end{aligned}
$$

It is clear that, from Eq. (20) and Eq. (13), the mean value and the stochastic fluctuation of overall CND interact each other. To obtain them one needs to study Eqs. (13) and (20) together, 
which is relatively complicated and difficult. If FIT can be simplified by simulation methods, then overall CND may be derived from Eq. (13) independently.

In our previous analyses (Qiao and Hong, 1998a, b), damage extent was assessed having little effect on crack-growth-rate. Hence, we assume that local crack-growth-rate is independent of time $t$ :

$A=A(c, \vec{x})$.

On the other hand, Eq. (13) implies that overall CND is not influenced by the stochastic fluctuation of local crack-nucleation-rate, because the crack-nucleation-rate is a linear term in the equilibrium equation. Therefore, the stochastic fluctuation of $n_{\mathrm{N}}(c, t, \vec{x})$ will be omitted in the following derivation. Note also that the crack-growth-rate tends to a constant level with the progress of fatigue process (Suh et al., 1992). Consequently, Eq. (13) reduces to:

$$
\begin{aligned}
& \frac{\partial \bar{n}(c, t)}{\partial t}+\frac{\partial[\bar{A}(c) \bar{n}(c, t)]}{\partial c} \\
& =N_{\mathrm{g}} \bar{n}_{\mathrm{N}}(c)-\overline{\left(\frac{\partial[\tilde{A}(c, \vec{x}) \tilde{n}(c, t, \vec{x})]}{\partial c}\right)} .
\end{aligned}
$$

The second term on the right side of above equation may be investigated by using the analytic solution of Eq. (2) (Ke et al., 1990):

$n(c, t, \vec{x})=\frac{1}{A(c, \vec{x})} \int_{\eta(c, t, \vec{x})}^{c} N_{\mathrm{g}} n_{\mathrm{N}}\left(c^{\prime}, \vec{x}\right) \mathrm{d} c^{\prime}$,

where the definition of lower integral boundary $\eta(c, t, \vec{x})$ is that for a crack with an initial length of $\eta(c, t, \vec{x})$ at $t=0$, its length will advance to $c$ at time $t$ under the growth rate of $A(c, \vec{x})$. Eq. (23) suggests that there is a saturation distribution in the evolution process of local CND, i.e. with the progress of the fatigue process, the distribution of local CND gradually tends to a local saturation curve from a small to a large value of crack length (also see Fig. 1). It is obvious that the saturation curve presents the stable distribution of CND. Referring to Eq. (23), we may write the local saturation curve as: $n_{0}(c, \vec{x})=\frac{1}{A(c, \vec{x})} \int_{0}^{c} N_{\mathrm{g}} n_{\mathrm{N}}\left(c^{\prime}, \vec{x}\right) \mathrm{d} c^{\prime}$.

At the stage that the fatigue damage fully developed, assume local CND be of the trend with its distribution approaching to the saturation curve. Letting the mean value and the stochastic fluctuation of saturation local CND be $\bar{n}_{0}(c)$ and $\tilde{n}_{0}(c, \vec{x})$, respectively, from Eq. (24), one may write

$\tilde{n}_{0}(c, \vec{x})=\tilde{B}(c, \vec{x}) \int_{0}^{c} N_{\mathrm{g}} n_{\mathrm{N}}\left(c^{\prime}\right) \mathrm{d} c^{\prime}$,

where $\tilde{B}(c, \vec{x})$ is the stochastic fluctuation of $1 / A(c, \vec{x})$. Then, we have

$\overline{\tilde{A}(c, \vec{x}) \tilde{n}_{0}(c, \vec{x})}=\overline{\tilde{A}(c, \vec{x}) \tilde{B}(c, \vec{x})} \int_{0}^{c} N_{\mathrm{g}} n_{\mathrm{N}}\left(c^{\prime}\right) \mathrm{d} c^{\prime}$,

where $\overline{\tilde{A}(c, \vec{x}) \tilde{n}_{0}(c, \vec{x})}$ is the mean value of $\tilde{A}(c, \vec{x}) \tilde{n}_{0}(c, \vec{x})$ and $\bar{A}(c, \vec{x}) \tilde{B}(c, \vec{x})$ is the mean value of $\tilde{A}(c, \vec{x}) \tilde{B}(c, \vec{x})$. Note that

$$
\begin{aligned}
& \overline{\tilde{A}(c, \vec{x}) \tilde{B}(c, \vec{x})}=\overline{\tilde{A}(c, \vec{x})\left(\frac{1}{\tilde{A}(c, \vec{x})}\right)}-\bar{A}(c) \overline{\left(\frac{1}{A(c, \vec{x})}\right)} \\
& =1-\bar{A}(c) \overline{\left(\frac{1}{A(c, \vec{x})}\right)} \text {. }
\end{aligned}
$$

Replacing local CND $n(c, t, \vec{x})$ with the saturation curve $n_{0}(c, \vec{x})$ (Eq. (24)) in Eqs. (26) and (27), and referring to Eq. (15), we can show FIT in the following form:

$$
\begin{aligned}
-\frac{\partial \mu}{\partial c}= & \frac{\partial}{\partial c}\left[\bar{A}(c) \overline{\left(\frac{1}{A(c, \vec{x})}\right)}\right] \int_{0}^{c} n_{\mathrm{N}}\left(c^{\prime}\right) \mathrm{d} c^{\prime} \\
& -n_{\mathrm{N}}(c)\left[1-\bar{A}(c) \overline{\left.\left(\frac{1}{A(c, \vec{x})}\right)\right]} .\right.
\end{aligned}
$$

Assume that the distribution of local crackgrowth-rate is a logarithm normal function, i.e. the probability for $A(c, \vec{x})=v$ is 


$$
f(v)= \begin{cases}\frac{1}{\sqrt{2 \pi} \sigma^{\prime}(c) v} \exp \left\{-\frac{\left[\ln v-\mu^{\prime}(c)\right]^{2}}{2 \sigma^{\prime 2}(c)}\right\} & (v \geqslant 0), \\ 0 & (v<0),\end{cases}
$$

where $\sigma^{\prime}(c)$ and $\mu^{\prime}(c)$ are distribution parameters determined by crack length $c$. The mean value of $f(v)$ is

$E(c)=\bar{A}(c)=\exp \left[\mu^{\prime}(c)+\frac{\sigma^{\prime 2}(c)}{2}\right]$

and the variance of $f(v)$ is

$D(c)=\exp \left[2 \mu^{\prime}(c)+\sigma^{\prime 2}(c)\right]\left\{\exp \left[\sigma^{\prime 2}(c)\right]-1\right\}$.

Through Eqs. (29) and (30), one derives

$$
\begin{aligned}
\overline{\left(\frac{1}{A(c, \vec{x})}\right)} & =\int_{0}^{\infty} \frac{1}{v} f(v) \mathrm{d} v \\
& =\exp \left[\frac{\sigma^{\prime 2}(c)}{2}-\mu^{\prime}(c)\right] \\
& =\frac{1}{\bar{A}(c)} \exp \left[\sigma^{\prime 2}(c)\right] .
\end{aligned}
$$

Substitution above equation into (28) gives:

$$
\begin{aligned}
-\frac{\partial \mu(c)}{\partial c}= & n_{\mathrm{N}}(c)\left[\mathrm{e}^{\sigma^{\prime 2}(c)}-1\right] \\
& +\left\{2 \sigma^{\prime}(c) \mathrm{e}^{\sigma^{\prime 2}(c)} \int_{0}^{c} n_{\mathrm{N}}\left(c^{\prime}\right) \mathrm{d} c^{\prime}\right\} \frac{\partial \sigma^{\prime}(c)}{\partial c} .
\end{aligned}
$$

Thus, the equilibrium equation of overall CND (Eq. (22)) can be solved independently by using Eq. (33). Here we assume that $\sigma^{\prime}(c)$ and $n_{\mathrm{N}}(c)$ have the following forms:

$\sigma^{\prime}(c)=\alpha \exp (\beta c)$

$n_{\mathrm{N}}(c)= \begin{cases}1-\frac{c}{2} & (c \leqslant 2), \\ 0 & (c>2),\end{cases}$

where $\alpha$ and $\beta$ are constants. Eq. (35) comes from previous experimental observations and it shows that smaller fatigue cracks nucleate easier (Qiao and Hong, 1998a).
Since the difference between stochastic analyses and mean-field theory comes from the term of FIT in Eq. (13), the following discussions will concentrate on the characteristics of FIT. Fig. 7 illustrates the tendency of FIT varying with different values of $c$ and $\beta$. It is seen that FIT is slightly larger than zero at the negative part of $\beta$-axis. If crack length $c$ is relatively large, there appears a negative peak of FIT at the positive end of the $\beta$-axis.

It is derived that $\sigma^{\prime}(c)$ as a function of $\bar{A}(c)$ and $D(c)$ from Eqs. (30) and (31):

$\sigma^{\prime}(c)=\sqrt{\ln \left[1+\frac{D(c)}{\bar{A}^{2}(c)}\right]}$.

Define a parameter $Q$ as

$Q=\frac{1}{2} \frac{\partial \ln [D(c)] / \partial c}{\partial \ln [\bar{A}(c)] / \partial c}$.

According to Eq. (37), we see that if $Q>1$, i.e. the variation extent of $D(c)$ is faster than that of $\vec{A}^{2}(c)$, the variation tendency of $\sigma^{\prime}(c)$ is of the same trend with that of $\bar{A}(c)$. Thus, because of the deceleration-acceleration pattern of short-fatigue-crack evolution (Fig. 8; Lankford, 1982), one may determine that in the short-crack regime $\partial \sigma^{\prime}(c) / \partial c<0$ $(\beta<0)$ and in the long-crack regime $\partial \sigma^{\prime}(c) / \partial c>0$ $(\beta>0)$. Therefore, in Fig. 7 , the process that $\beta$ varies from -1 to 1 corresponds to the process

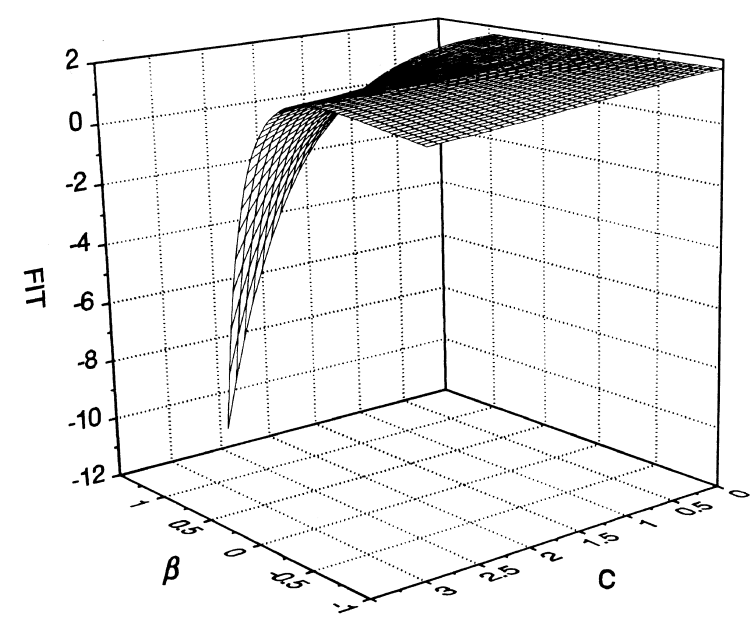

Fig. 7. Variation of FIT as a function of $c$ and $\beta$. 


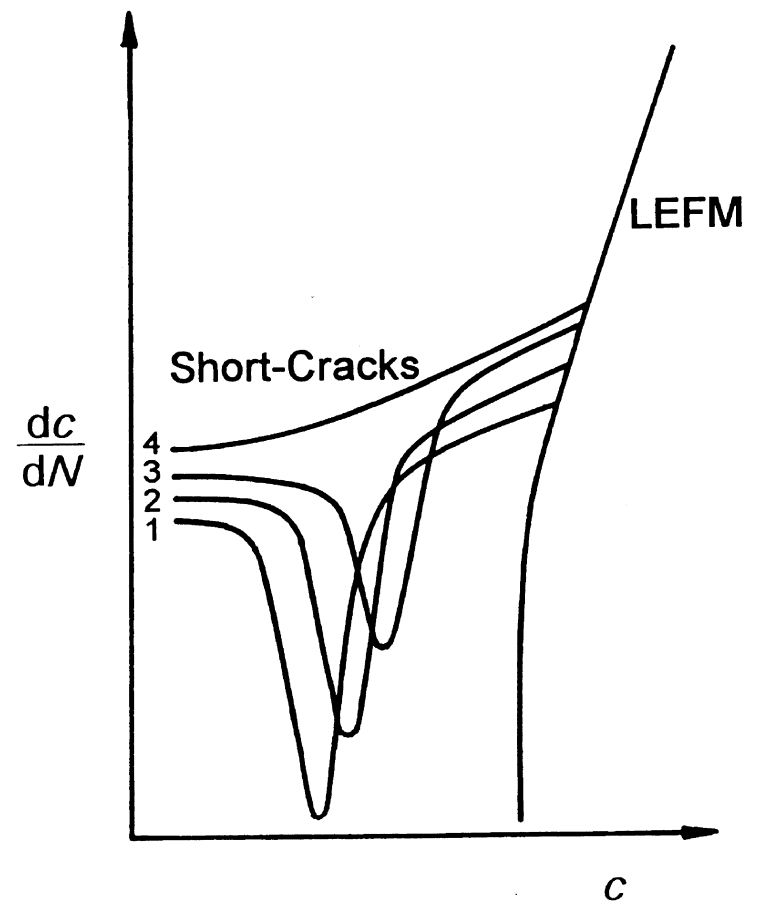

Fig. 8. Deceleration-acceleration pattern of short-fatigue-crack propagation. (Grain $1<$ Grain $2<$ Grain $3 \ll$ Grain 4.)

that fatigue damage develops from the short-crack regime into the long-crack regime.

At the initial stage of the fatigue process, fatigue damage is within the short-crack regime, thus FIT is at the negative part of $\beta$-axis. From Fig. 7, it is observed that FIT is somewhat larger than zero, which implies that the result of stochastic analyses should be slightly larger than the result of mean-field theory. With the progress of the fatigue process, dispersed fatigue-cracks develop into long-crack regime, thus FIT develops to the positive part of $\beta$-axis, where the negative peak of FIT in the region of large values of crack length $c$ may intensively suppress the propagation of cracks. Therefore, at the end of the short-crack regime and in the whole long-crack regime, the damage extent derived by stochastic analyses may be much weaker than by mean-field theory. The above discussion interprets the difference between stochastic analyses and mean-field theory shown in Fig. 4, in which the data points represent random processes with $Q>1$.

\section{Conclusions}

By integrating the equilibrium equation of local $\mathrm{CND}$, the equilibrium equation of overall CND is obtained and the following conclusions are drawn:

(1) Overall damage evolution is influenced by the stochastic fluctuation of the local crackgrowth-rate. The influence extent may be described by the standard errors of local crack-growth-rate and local CND, and their correlation coefficient.

(2) The difference between stochastic analyses and mean-field theory comes from the fluctuation influence term related to the stochastic fluctuation of crack-growth-rate. Overall damage evolution is independent of the stochastic fluctuation of cracknucleation-rate.

(3) At the initial stage of fatigue process, the damage extent derived by stochastic analyses is slightly larger than that derived by mean-field theory and at the following stage of fatigue process the result of stochastic analyses is smaller than that of mean field theory. The tendency of simulation results by stochastic analyses is consistent with experimental measurements.

\section{Acknowledgements}

This paper was supported by the National Outstanding Youth Scientific Award of China, the National Natural Science Foundation of China and the Chines Academy of Sciences.

\section{References}

Fang, B., Hong, Y.S., Bai, Y.L., 1995. Experimental and theoretical study on numerical density evolution of short fatigue cracks. Acta Mechanica Sinica (English edition) 11, $144-152$

Hong, Y.S., Gu, Z.Y., Fang, B., Bai, Y.L., 1997. Collective evolution characteristics and computer simulation of short fatigue cracks. Phil. Mag. A 75, 1517-1531.

Hong, Y.S., Lu, Y.H., Zheng, Z.M., 1989. Initiation and propagation of a short fatigue crack in a weld metal. Fatigue Fract. Engrg. Mater. Struct. 12, 323-331.

Hong, Y.S., Lu, Y.H., Zheng, Z.M., 1991. Orientation preference and fractal character of short fatigue cracks in a weld metal. J. Mater. Sci. 26, 1821-1826. 
Hong, Y.S., Qiao, Y., 1998. Analysis of damage moments in the collective evolution of short fatigue cracks. Key Engrg. Mater. 145-149, 399-404.

Hong, Y.S., Qiao, Y., Liu, N., Zheng, X.H., 1998. Effect of grain size on collective damage of short fatigue cracks and fatigue life estimation for a stainless steel. Fatigue Fract. Engrg. Mater. Struct. 21, 1317-1325.

Hong, Y.S., Zheng, X.H., Liu, N., 1999. An experimental investigation on collective damage of short fatigue cracks for a stainless steel. Proceedings of ICM8, Progress in Mechanical Behaviour of Materials. Vol. 1, p. 53-56.

Hussain, K., de los Rios, E.R., Navarro, A.A., 1993. Two-stage micromechanics model for short fatigue cracks. Engrg. Fract. Mech. 44, 425-436.

Ke, F.J., Bai, Y.L., Xia, M.F., 1990. Evolution of ideal microcrack system. Science in China A 6, 621-631 (in Chinese).
Lankford, J., 1982. The growth of small fatigue cracks in 7076-T6 Aluminium. Fatigue Engrg. Mater. Struct. 5, 233-248

Price, C.E., 1988. The progression of bending fatigue in Nickel. Fatigue Fract. Engrg. Mater. Struct. 11, 483-492.

Qiao, Y., Hong, Y.S., 1998a. An analysis of collective damage for short fatigue cracks based on equilibrium of crack numerical density. Engrg. Fract. Mech. 59, 151-163.

Qiao, Y., Hong, Y.S., 1998b. A stochastic model for evolution of collective short-fatigue-cracks based on local field analysis. Acta Mechanica Sinica 30, 564-571 (in Chinese).

Suh, C.M., Lee, J.J., Kang, Y.G., Ahn, H.J., Woo, B.C., 1992. A simulation of the fatigue crack process in type 304 stainless steel at $538^{\circ} \mathrm{C}$. Fatigue Fract. Engrg. Mater. Struct. 15, 671-684. 